\title{
Mutual Compensation of Threshold Voltage and Thermal Voltage Temperature Effects with Applications in Voltage Reference Generator
}

\author{
Jiangxu WU ${ }^{1, a}$, Jianguo $\mathrm{HU}^{2, \mathrm{~b}, *}$, Zhikui DUAN ${ }^{3}$ \\ ${ }^{1}$ School of Electronics and Information Technology \\ Sun Yat-Sen University \\ Guangzhou, China \\ ${ }^{2}$ Sun Yat-Sen University \\ Guangzhou, China \\ ${ }^{3}$ Foshan University \\ Guangzhou, China \\ E-mail: ${ }^{a}$ wujx27@mail2.sysu.edu.cn \\ *E-mail: bhujianguo@mail.sysu.edu.cn \\ +* Corresponding author
}

\begin{abstract}
A low power, low temperature coefficient (TC) CMOS voltage reference generator is proposed in this paper, which exploits temperature mutual compensation relationship between threshold voltage and thermal voltage, and provides a mean reference voltage of $411.8 \mathrm{mV}$.In this reference, a proportional to absolute temperature (PTAT) current containing the thermal voltage which has a positive $T C$ is generated, and then it is injected into a diode-connected NMOS transistor that supply the threshold voltage which has a negative TC. The mixing of the two voltages produces a reference voltage with zero $\mathrm{TC}$. The proposed circuit is verified by SPICE simulation with CMOS 0.18 um process technology. The simulation results show that the power consumption is $17.9 \mathrm{nW}$ and the $\mathrm{TC}$ is $8.6 \mathrm{ppm} /{ }^{\circ} \mathrm{C}$. The proposed circuit is suitable for power sensitive applications.
\end{abstract}

Keywords-voltage reference; low power; low tc; cmos; thermal voltage

\section{INTRODUCTION}

RFID tag are used numerous in medical field, they are attached to the medical apparatus and instruments to register, track and monitor them [1]. Tags used in the medical field need to be able to work under high temperature, because these devices like surgical instruments should be sterilized by high temperature and high pressure with a temperature about 121 134 degrees. Meanwhile, Passive systems require lower power consumption. For this purpose, many kinds of voltage reference circuits are proposed. For example, some advanced bandgap voltage reference based on parasitic bipolar transistor have lower power consumption than the traditional bandgap reference are invented [2, 3], but they are not performs satisfactorily because of the parasitic bipolar transistor in a standard CMOS process is usually not very well characterized. Meanwhile, many nanopower voltage reference circuits based on the fact that the threshold voltage of MOSFETs with different gate oxide thickness in the same CMOS technology exhibit different temperature characteristic are proposed $[4,5,6,7]$, but such solutions also can not be implemented in a standard CMOS technology because they require additional fabrication steps. Besides, a novel voltage reference was introduced in $[8,9,10]$, these devices consist of standard MOSFETs operating in subthreshold regime. It generates a MOSFET threshold voltage with negative TC and a multiple of the thermal voltage with positive TC, and then adds them to produce a voltage reference with zero TC, but they either have high TC [8], or have high power consumption [9], or both have [10]. Besides it can not applicable at high temperature $[9,10]$.

To solve the above mentioned problems, a novel voltage reference circuit is presented in this paper. It generates a PTAT current containing the thermal voltage with a positive TC and then injected into a diode-connected NMOS transistor which supplies the threshold voltage with a negative TC to achieve an output voltage reference near zero TC. Simulation results show that this work has low power dissipation, low TC and high temperature.

\section{PROPOSED CIRCUIT}

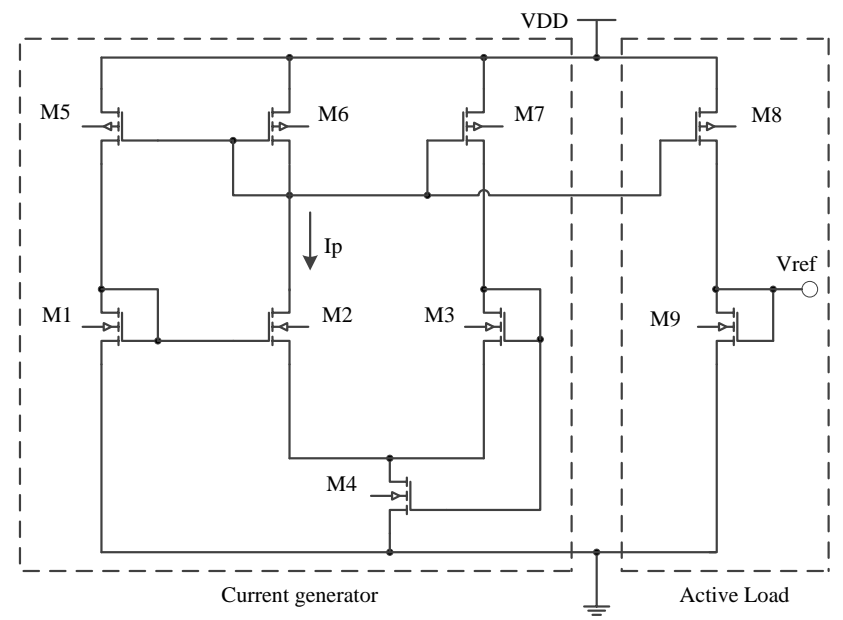

Figure 1. Proposed voltage reference circuit

The architecture of the proposed voltage reference is shown in Fig. 1. It consists of a current generator and an active load. Start-up circuit is ignored here. The current generator generates a PTAT current $I_{P}$, and then injected 
into the active load that include a diode-connected transistor to generate the reference voltage $V_{\text {ref }}$. All the MOSFETs except for M3, M4 and M9 are operated in the subthreshold region. The MOSFET M4 is operated in the strong-inversion and deep triode region, and the MOSFET M3 and M9 are operated in the saturation.

\section{A. Current Generator Circuit}

The current-voltage characteristics of a MOSFET that operates in the subthreshold region can be approximated by Eq. (1).

$$
I_{D}=K I_{0} \exp \left(\frac{V_{G S}-V_{T H}}{m V_{T}}\right) \times\left[1-\exp \left(-\frac{V_{D S}}{V_{T}}\right)\right]
$$

where $K$ is the aspect ratio $\left(=\frac{W}{L}\right.$ ) of the transistor ( $W$ and $L$ are the channel width and length), $V_{G S}$ and $V_{D S}$ are the gate-source voltage and the drain- source voltage, respectively. $I_{0}=\mu C_{o x} \frac{W}{L}(m-1) V_{T}^{2}, \mu$ is the electron mobility in the channel, $C_{o x}$ is the gate-oxide capacitance, $V_{T}=\frac{K_{B} T}{q}$ ) is the thermal voltage $\left(K_{B}\right.$ is the Boltzmann constant, $T$ is the absolute temperature, and $q$ is the elementary charge), $V_{T H}$ is the threshold voltage, and $m$ is the subthreshold slope factor.

The current generator can generate the current $I_{P}$ without using a resistance. This is extremely important if the current $I_{P}$ need to be very small, to drastically reduce the power consumption of the circuit. In such case a large resistance would be required causing a large area occupation on the chip. The channel length modulation effect of M4 is negligible since it is long channel devices. Besides, the drain-source voltage in M4 is very small by setting the parameter, thus the MOSFETs M1 and M2 have larger drain-source voltage than their thermal voltage. As $V_{D S} \geq 0.1 V$, current $I_{D}$ is almost independent of $V_{D S}$ and given by Eq. (2)[9].

$$
I_{D}=K I_{0} \exp \left(\frac{V_{G S}-V_{T H}}{m V_{T}}\right)
$$

The current $I_{P}$ can be derived through the following steps. From Fig. 1 the drain-source voltage $V_{D S 4}$ is given by Eq. (3).

$$
V_{D S 4}=V_{\mathrm{GS} 1}-V_{G S 2}
$$

From Eq. (2), we arrive at the expression:

$$
V_{G S}=V_{T H}+m V_{T} \ln \left(\frac{I_{D}}{K I_{0}}\right)
$$

Because the transistor M5 and M6 consist of current mirror, the current in M1 is $\frac{K_{5}}{K_{6}} I_{P}$. Therefore, the voltage $V_{D S 4}$ can be expressed as:

$$
V_{D S 4}=m V_{T} \ln \left(\frac{K_{5} K_{2}}{K_{6} K_{1}}\right)
$$

The channel length between transistors pairs (M5-M8, M1-M2, and M3-M4) are designed equally to reduce the threshold voltage mismatch.

Due to M3 operated in saturation and M4 operated in a strong-inversion, deep-triode region, the drain current in M3 and M4 can be expressed as:

$$
I_{3}=\frac{1}{2} \mu_{n} C_{o x} K_{3}\left(V_{G S 3}-V_{T H}\right)^{2}
$$

$$
I_{4}=\mu_{n} C_{o x} K_{4}\left(V_{G S 3}-V_{T H}+\frac{1}{2} V_{D S 4}\right) V_{D S 4}
$$

The MOSFET M7 copy the current $I_{P}$ in proportion from M6, consequently the current in M3 is $\frac{K_{7}}{K_{6}} I_{P}$, and the current in M4 is the sum of the current in M2 and M4. Rewrite the above two expressions we have:

$$
\frac{K_{7}}{K_{6}} I_{P}=\frac{1}{2} \mu_{n} C_{o x} K_{3}\left(V_{G S 3}-V_{T H}\right)^{2}
$$

$$
\left(1+\frac{K_{7}}{K_{6}}\right) I_{P}=\mu_{n} C_{o x} K_{4}\left(V_{G S 3}-V_{T H}+\frac{1}{2} V_{D S 4}\right) V_{D S 4}
$$

Combine the Eq. (8) and Eq. (9), we can obtain that:

$$
V_{G S 3}-V_{T H}=K+\sqrt{K+K^{2}} V_{D S 4}
$$




$$
\text { where } K=\frac{K_{4} K_{7}}{K_{3}\left(K_{6}+K_{7}\right)} \text {. Then substituting Eq. }
$$
and Eq. (10) in Eq. (8), the PTAT current $I_{P}$ is obtained as:

$$
I_{P}=\frac{1}{2} \mu_{n} C_{o x} \frac{K_{3} K_{6}}{K_{7}}\left(K+\sqrt{K+K^{2}}\right)^{2} m^{2} V_{\mathrm{T}}^{2} \ln ^{2}\left(\frac{K_{5} K_{2}}{K_{6} K_{1}}\right)
$$

From the Eq. (11), it can be seen that a current contains the thermal voltage is obtained, we can inject it into a diode-connected transistor to compensate the threshold voltage, then a zero TC reference voltage can be generated.

\section{B. Active Load}

Because the diode-connected transistor M9 working in the saturation region, the reference voltage can be expressed as:

$$
V_{r e f}=V_{t h 4}+\sqrt{\frac{2 I_{9}}{\mu_{n} C_{o x} K_{4}}}
$$

The generated current $I_{P}$ is mirrored into a diode-connected transistor M9. The current in M9 can be expressed as $I_{9}=\frac{K_{8}}{K_{6}} I_{P}$, thus the Eq. (12) becomes:

$$
V_{r \sigma f}=V_{t h 4}+\sqrt{\frac{2 K_{8}}{K_{6} K_{4}} \frac{I_{P}}{\mu_{n} C_{\alpha x} K_{4}}}
$$

Substituting Eq. (11) in Eq. (13), and the reference voltage $V_{\text {ref }}$ is given by:

$$
\begin{gathered}
V_{r e f}=V_{t h 4}+\sqrt{\frac{K_{8} K_{3}}{K_{7} K_{4}}}\left(K+\sqrt{K+K^{2}}\right) m V_{T} \ln \left(\frac{K_{5} K_{2}}{K_{6} K_{1}}\right) \\
\text { where } \\
K=\frac{K_{4} K_{7}}{K_{3}\left(K_{6}+K_{7}\right)} .
\end{gathered}
$$

\section{Temperature Compensation}

From the Eq. (13), we can see that $V_{\text {ref }}$ consists by $V_{\text {th } 4}$ which has a negative TC and $V_{T}$ which has a positive TC. So a zero TC reference voltage can be obtained by adjusting the coefficients in front of $V_{T}$.

The temperature dependence of the threshold voltage can be given by:

$$
V_{T H}(T)=V_{T H}\left(T_{0}\right)+\alpha\left(T-T_{0}\right)
$$

where $T_{0}$ is the reference temperature which is $300^{\circ} \mathrm{K}$ and $\alpha$ is a negative value [11], substituting Eq. (15) and $V_{T}=\frac{K_{B}}{q}$ in Eq. (14), the reference voltage $V_{\text {ref }}$ can be rewritten as:

$V_{r e f}=V_{t h 4}\left(T_{0}\right)+\alpha\left(T-T_{0}\right)+m \frac{K_{B} T}{q} \sqrt{\frac{K_{8} K_{3}}{K_{7} K_{4}}}\left(K+\sqrt{K+K^{2}}\right) \ln \left(\frac{K_{5} K_{2}}{K_{6} K_{1}}\right)$

By differentiating Eq. (16) with respect to the temperature, we obtain:

$$
\frac{\partial V_{r e f}}{\partial T}=\alpha+m \frac{K_{B}}{q} \sqrt{\frac{K_{8} K_{3}}{K_{7} K_{4}}}\left(K+\sqrt{K+K^{2}}\right) \ln \left(\frac{K_{5} K_{2}}{K_{6} K_{1}}\right)
$$

By setting Eq. (17) to zero, we obtain the condition:

$$
-\alpha=m \frac{K_{B}}{q} \sqrt{\frac{K_{8} K_{3}}{K_{7} K_{4}}}\left(K+\sqrt{K+K^{2}}\right) \ln \left(\frac{K_{5} K_{2}}{K_{6} K_{1}}\right)
$$

Therefore, if Eq. (18) is satisfied, a zero TC reference voltage can be obtained. From Eq. (16) and Eq. (18), the reference voltage $V_{\text {ref }}$ is expressed as:

$$
V_{\text {ref }}=V_{T H 4}\left(T_{0}\right)
$$

therefore, the circuit generates the threshold voltage of MOSFET at absolute zero temperature.

\section{Simulation Results}

To verify the performance of the proposed design, we use the SPICE simulator to simulate the circuit with SMIC 0.18um CMOS process. Fig. 2 shows a plot of the measured output voltages versus temperature with a range from $0^{\circ} \mathrm{C}$ to $150^{\circ} \mathrm{C}$, the calculated $\mathrm{TC}$ is $8.6 \mathrm{ppm} /{ }^{\circ} \mathrm{C}$ at $\mathrm{VDD}=0.7 \mathrm{~V}$. Fig. 3 shows the voltage reference $V_{\text {ref }}$ as a function of power supply from $0 \mathrm{~V}$ to $1.8 \mathrm{~V}$ at room temperature, with a supply line sensitivity (LS) of about $1.96 \% / V$. Fig. 4 shows the simulation result of PSRR of the voltage reference $V_{\text {ref }}$ when $\mathrm{T}=20^{\circ} \mathrm{C}$ and $\mathrm{VDD}=0.7 \mathrm{~V}$. The PSRR without any filtering capacitor is $-80 \mathrm{~dB}$ at $100 \mathrm{~Hz}$ and $-51.7 \mathrm{~dB}$ at $10 \mathrm{MHz}$, therefore the proposed circuit has a better PSRR performance. Fig. 5 shows the proposed circuit generates a mean reference voltage of about $415.7 \mathrm{mV}$ with a variation of $3.9 \mathrm{mV}$ at four different power supply voltage: $0.7 \mathrm{~V}, 1 \mathrm{~V}$, $1.4 \mathrm{~V}$, and $1.8 \mathrm{~V}$. Fig. 6 shows the reference $V_{\text {ref }}$ at all corners. Fig. 7 shows the TC from $0^{\circ} \mathrm{C}$ to $150^{\circ} \mathrm{C}$ at all corners, the TC of the TT corner is $8.6 \mathrm{ppm} /{ }^{\circ} \mathrm{C}$, and those of the FF, FS, SF and SS corners are $22.2 \mathrm{ppm} /{ }^{\circ} \mathrm{C}, 8.9 \mathrm{ppm} /{ }^{\circ} \mathrm{C}$, $14.7 \mathrm{ppm} /{ }^{\circ} \mathrm{C}$ and $35.7 \mathrm{ppm} /{ }^{\circ} \mathrm{C}$, respectively. Fig. 8 shows 
the measured power consumption across temperature, the results shows the power increases by $4 \mathrm{~mW}$ for every 10 degrees increase in temperature. Fig. 9 shows the measured power consumption across supply voltage. The total power consumption of the proposed circuit is $17.9 \mathrm{nW}$ at $0.7 \mathrm{~V}$ power supply and at room temperature. To verify the stability of the circuit operation with process variations. Monte Carlo analysis assuming WID mismatch variations in all MOSFETs were considered. Fig. 10 shows the distribution of average output voltage $V_{\text {ref }}$ at $0.7 \mathrm{~V}$ power supply and room temperature. We assumed a Gaussian distribution $\left(\delta_{v t h}\right)$ for the WID variation. The average voltage $V_{\text {ref }}$ was about $411.7012 \mathrm{mV}$ in this simulation. The coefficient of variation ( $\delta / \mu$ ) in 270 runs was $0.27 \%$. Table I summarize the main performance of the proposed circuit and compares with previous works. As this table reveals, the proposed circuit operates with low TC, low power consumption and high PSRR.

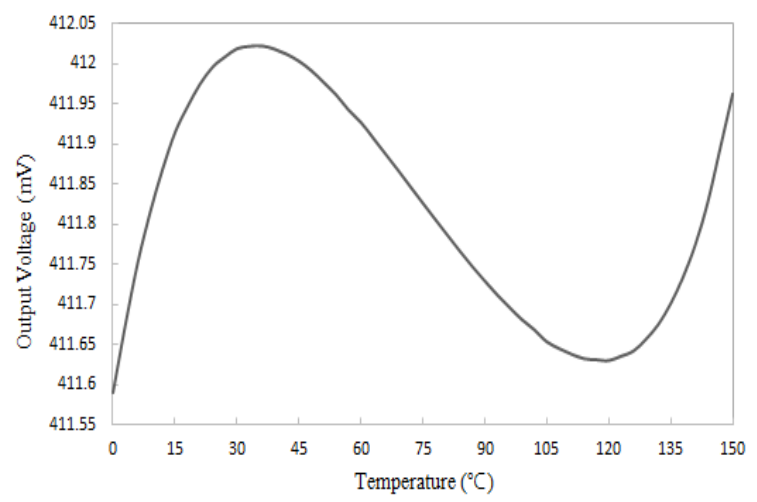

Figure 2. The output reference voltage $V_{\text {ref }}$ versus temperature

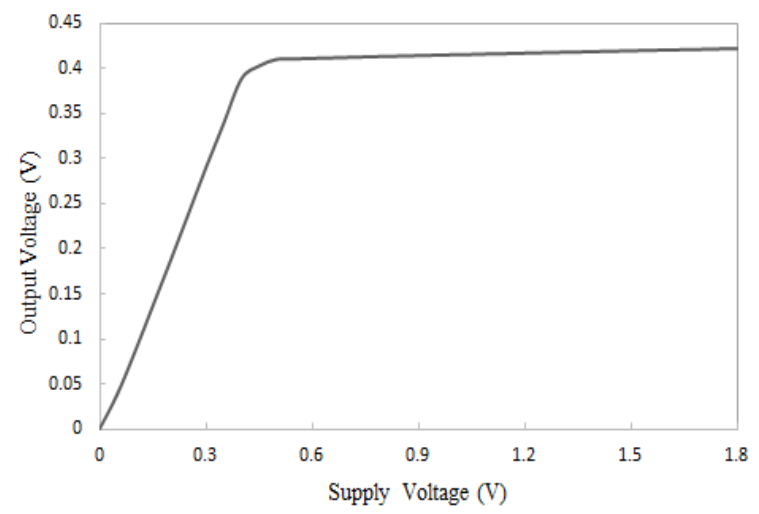

Figure 3. The output reference voltage $V_{\text {ref }}$ versus supply voltage

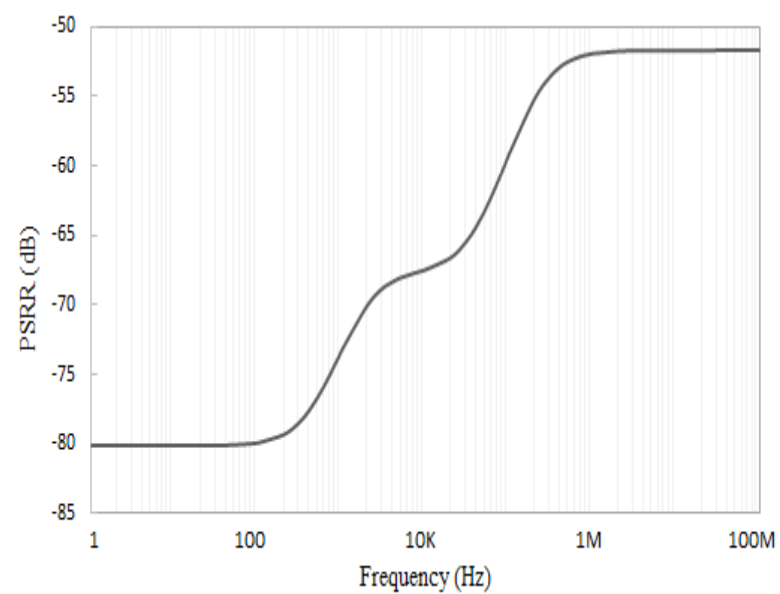

Figure 4. The PSRR at room temperature and a $0.7 \mathrm{~V}$ supply power

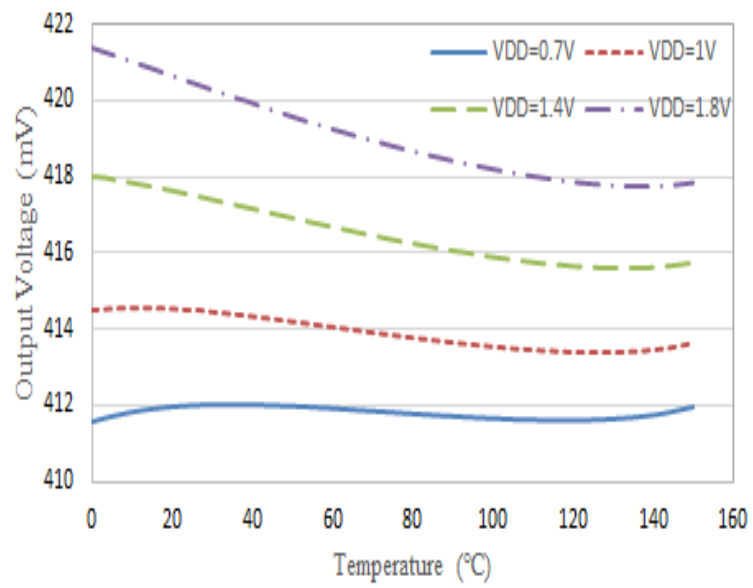
Figure 5. The output voltage reference
temperature at different

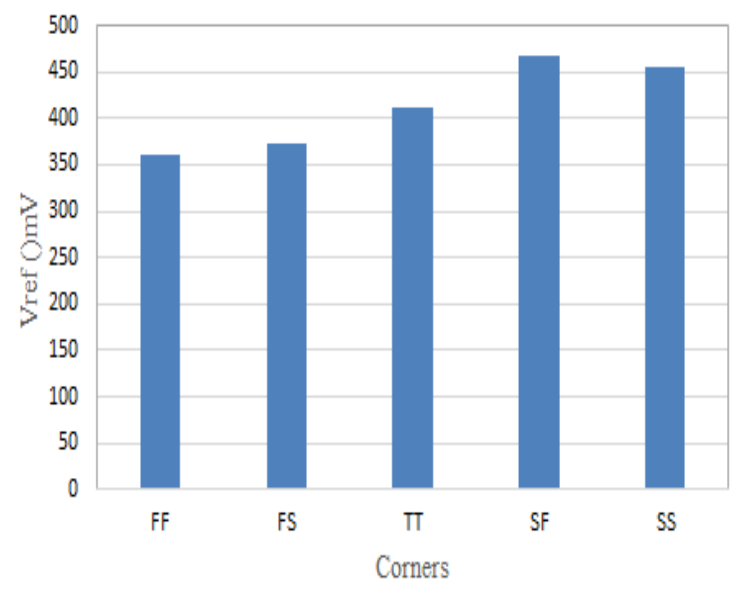

Figure 6. $\quad$ Measured $V_{\text {ref }}$ at all corners 


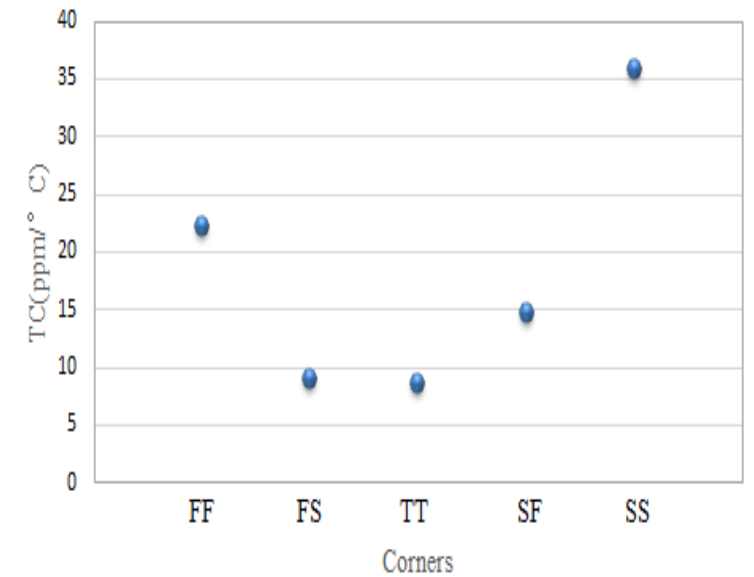

Figure 7. Measured TC at all corners

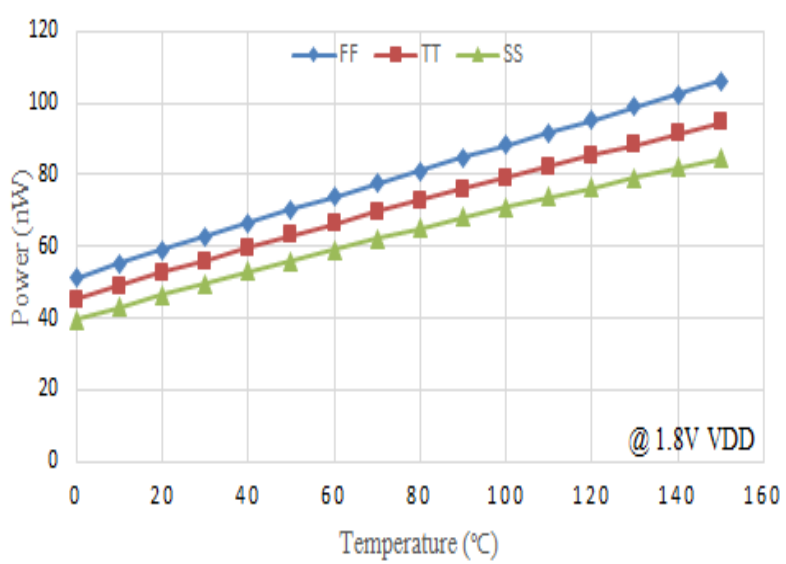

Figure 8. Measured power across temperature

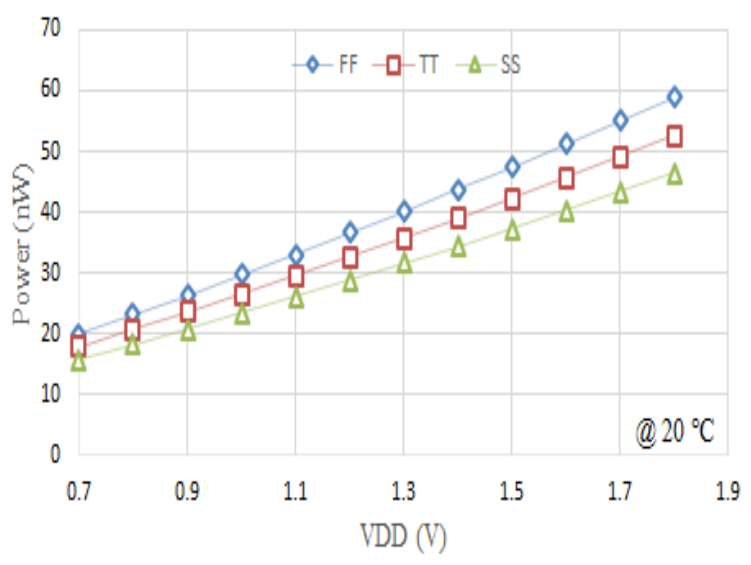

Figure 9. Measured power across VDD

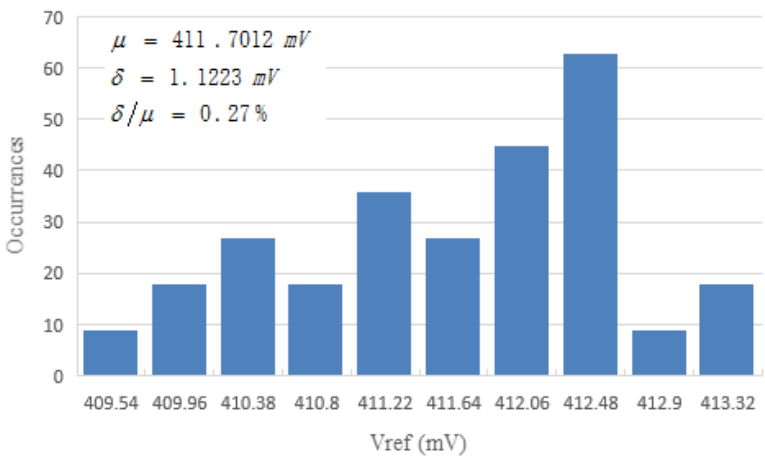

Figure 10. Fig. 10. Distribution of output voltage for 270-point Monte Carlo simulations assuming WID variations

TABLE I. PERFORMANCE COMPARISON

\begin{tabular}{|c|c|c|c|c|c|}
\hline & This work & [7] & [8] & [9] & [10] \\
\hline Process & $0.18 \mathrm{um}$ & $0.18 \mathrm{um}$ & $0.18 \mathrm{um}$ & $0.35 \mathrm{um}$ & $0.18 \mathrm{um}$ \\
\hline $\begin{array}{c}\text { Temp range } \\
\left({ }^{\circ} \mathrm{C}\right)\end{array}$ & $0 \sim 150$ & $0 \sim 125$ & $-10 \sim 150$ & $-20 \sim 80$ & $-40 \sim 120$ \\
\hline $\mathrm{VDD}(\mathrm{V})$ & $0.7 \sim 1.8$ & $0.45 \sim 2$ & $0.6 \sim 2.2$ & $1.4 \sim 4$ & $1 \sim 3$ \\
\hline Vref(mV) & 411.8 & 263.5 & 650 & 745 & 610 \\
\hline Power(nW) & 17.9@Room temp & 2.6@Room temp & 5.1@ Room temp & $\begin{array}{c}300 @ \text { Room } \\
\text { temp }\end{array}$ & $\begin{array}{c}230 @ \text { Room } \\
\text { temp }\end{array}$ \\
\hline $\mathrm{TC}\left(\mathrm{ppm} /{ }^{\circ} \mathrm{C}\right)$ & 8.6 & 142 & 37 & 7 & 66.9 \\
\hline \multirow[t]{2}{*}{ PSRR } & $-80 \mathrm{~dB} @ 100 \mathrm{~Hz}$ & $-65 \mathrm{~dB} @ 100 \mathrm{~Hz}$ & $-40 \mathrm{~dB} @ 1 \mathrm{KHz}$ & $-45 \mathrm{~dB}$ & $-15.45 \mathrm{~dB}$ \\
\hline & $-51.7 \mathrm{~dB} @ 10 \mathrm{MHz}$ & -50dB@1KHz & -62dB@10MHz & @ $100 \mathrm{~Hz}$ & @10MHz \\
\hline
\end{tabular}




\section{CONCLUSION}

Our study presents a novel approach for low power, low $\mathrm{TC}$ and high temperature voltage reference. The design conditions to minimize the power consumption and the TC have been described in detail. The voltage reference has been implemented in SMIC 0.18-um CMOS process. The results of simulation show that a TC of $8.6 \mathrm{ppm} /{ }^{\circ} \mathrm{C}$ from $0^{\circ} \mathrm{C}$ to $150^{\circ} \mathrm{C}$, and power consumption of $17.9 \mathrm{nW}$ are achieved with a supply voltage of $0.7 \mathrm{~V}$. It indicates that the circuit can realize low power and low TC at the same time. Obviously the proposed circuit is suitable for power sensitive large scale integrated circuits such as portable mobile devices, life-assist medical devices and smart sensor. Meanwhile this circuit also suitable for high temperature working environment, like RFID tags in the medical field.

\section{REFERENCES}

[1] Cai-Feng Liu, Yi-Ming Gu: The research of intelligent medical devices management system based on RFID technology, ISAI (2016) 29-33.

[2] Ali Far: A 220nA bandgap reference with 80dB PSRR targeting energy harvesting, CCECE (2016) 1-4.

[3] K. Sanborn, Donsheng Ma, and V. Ivanov, A Sub-1-V low noise bandgap voltage reference, IEEE J. Solid-State Circuits 42 (2007) 2466-2481.

[4] Yunling Luo, Jun Zhang, Qiaobo Wang, Yanhang Zeng, Jianguo Hu and Hong-Zhou Tan: An ultra-low power CMOS subthreshold voltage reference, EDSSC (2012) 1-3.

[5] Z. Yang and M. Jiang: Nanopower CMOS voltage reference circuit with $16 \mathrm{ppm} /{ }^{\circ} \mathrm{C}$ from $0^{\circ} \mathrm{C}$ to $150^{\circ} \mathrm{C}$ without resistors, ICCE_TW (2015) 424-425.

[6] Qing Ding, Pengpeng Yuan, Dongmei Li and Zhihua Wang: A sub-1-V ultra-low power full CMOS bandgap reference working in subthreshold region, EDSSC (2014) 1-2.

[7] Luca Magnelli, Felice Crupi, Pasquale Corsonello and Calogero Pace, A $2.6 \mathrm{nW}, 0.45 \mathrm{~V}$ temperature-compensated subthreshold CMOS voltage reference, IEEE J. Solid-State Circuits 46 (2011) 465-474.

[8] Zhangcai Huang, Qin Luo and Yasuaki Inoue: A CMOS Sub-1-V nanopower current and voltage reference with leakage compensation, ISCAS (2010) 4069-4072.

[9] Ken Ueno, Tetsuya Hirose, Tetsuya Asai and Yoshihito Amemiya, A $300 \mathrm{nW}, 15 \mathrm{ppm} /{ }^{\circ} \mathrm{C}, 20 \mathrm{ppm} / \mathrm{V}$ CMOS voltage reference circuit consisting of subthreshold MOSFETs, IEEE J. Solid-State Circuits 44 (2009) 2047-2054.

[10] Jian Li, Jiancheng Li and Li Yang: A nanopower, high PSRR full CMOS voltage reference circuit consisting of subthreshold MOSFETs, ASICON (2015) 1-4.

[11] M. Filanovsky and A. Allam, Mutual compensation of mobility and threshold voltage temperature effects with application in CMOS circuits, IEEE Transaction on Circuits and Systems I: Fundamental Theory and Applications 48 (2001) 876-884. 